\title{
Gina Lagorio, Parlare al femminile
}

Gina Lagorio, Speaking in the Feminine

Gina Lagorio, Parler au féminin

Marie Louise Crippa

(2) OpenEdition

Journals

Edizione digitale

URL: https://journals.openedition.org/cei/8652

DOI: 10.4000/cei.8652

ISSN: 2260-779X

\section{Editore}

UGA Éditions/Université Grenoble Alpes

\section{Edizione cartacea}

ISBN: 978-2-37747-257-4

ISSN: $1770-9571$

\section{Notizia bibliografica digitale}

Marie Louise Crippa, «Gina Lagorio, Parlare al femminile», Cahiers d'études italiennes [Online], 32 | 2021 online dal 01 mars 2021, consultato il 16 septembre 2021. URL: http://journals.openedition.org/cei/ 8652 ; DOI: https://doi.org/10.4000/cei.8652

Questo documento è stato generato automaticamente il 16 septembre 2021

(c) ELLUG 


\title{
Gina Lagorio, Parlare al femminile
}

\author{
Gina Lagorio, Speaking in the Feminine \\ Gina Lagorio, Parler au féminin
}

Marie Louise Crippa

1 Il Novecento è considerato, per eccellenza, il secolo dell'emancipazione femminile: la donna esce dal focolare domestico per entrare finalmente nel mondo del lavoro, delle scienze, della politica, e fa delle arti un mestiere, non più solo un hobby.

Il processo, però, non è stato né facile dal punto di vista socio-politico, né uniforme dal punto di vista geografico: basti pensare alla particolare situazione italiana, dove l'arretratezza delle strutture economiche, le resistenze culturali opposte dalla Chiesa cattolica ed infine l'ostruzionismo fascista hanno reso il percorso più lento e difficoltoso rispetto ai più avanzati paesi europei. La lunga marcia delle donne italiane è iniziata nel tardo Ottocento, ha raggiunto piccole e grandi vittorie, non senza alcune contraddizioni e, forse, non si è ancora conclusa. Nel 1875 viene legalmente riconosciuto alle donne il diritto di accedere agli studi universitari, ma solo dopo quasi 90 anni, nel 1963, viene concessa loro la possibilità di ricoprire tutti gli incarichi pubblici'. Dal 1945 le donne possono esercitare il proprio diritto di voto, ma solo dopo trent'anni saranno dichiarate autonome dalla patria potestà del marito. Nel 1970 viene introdotto il divorzio ed otto anni più tardi l'aborto. Nel 1977 il parlamento si interroga per la prima volta sulla parità tra uomini e donne in materia di lavoro, ma solo venti anni dopo la violenza sessuale è considerata un delitto contro la persona e non più contro la moralità pubblica.

3 È in questo contesto che si inserisce il ritratto di Gina Lagorio, una delle rappresentanti più significative dell'emancipazione femminile italiana: impegnata fin dalla sua gioventù sul fronte e politico e culturale. Il suo nome è stato associato alla Resistenza Italiana e al movimento politico della Sinistra Indipendente, a istituzioni artistiche e letterarie, prima fra tutte la casa editrice Garzanti, per la quale ha curato diversi volumi in veste di editor e pubblicato molti testi in qualità di saggista e scrittrice. Insieme alle conquiste, Gina Lagorio ha rappresentato le complicazioni e le contraddizioni proprie della condizione femminile moderna. Le sue opere narrative 
denunciano, sotto vesti più o meno allegoriche, una tensione costante fra dimensione pubblica e privata, ragioni letterarie e spirito divulgativo, aspirazioni artistiche e doveri familiari. I suoi interventi sui giornali e in televisione hanno riserbato grande spazio alle tematiche sociali e culturali della donna, basti ricordare le rubriche Singolare femminile e Parlare al femminile, trasmesse sui canali della Radiotelevisione Italiana. I diritti delle donne e della famiglia sono stati obiettivi primari delle sue battaglie politiche, come attestano le dichiarazioni tenute in veste di Deputata e sui bollettini del PCI. I suoi scambi epistolari, amicali ed anche professionali, portano inscritta la testimonianza diretta di donna che non ha voluto rinunciare al suo ruolo di intellettuale, moglie e madre.

4 Il Centro Apice (Archivi della Parola, dell'Immagine e della Comunicazione Editoriale) conserva l'archivio personale di Gina Lagorio, nel quale, oltre a bozze dei suoi libri ed appunti di lavoro, è sopravvissuto un ricco epistolario: lettere intercorse con altre protagoniste dell'universo intellettuale, come Giovanna Bemporad ${ }^{2}$, Alba de Céspedes ${ }^{3}$, Marisa Fenoglio ${ }^{4}$, Cesira Fiori ${ }^{5}$, Giovanna Ioli ${ }^{6}$, Natalia Ginzburg ${ }^{7}$, Anna Maria Ortese ${ }^{8}$. Documenti inediti di carattere privato ed informale dove convivono riflessioni sull'attualità, considerazioni letterarie, pianificazioni editoriali, ma anche aneddoti personali, gioie e fatiche della vita familiare, toni intimistici e temi minori tradizionalmente esclusi dalla dimensione intellettuale.

5 Luigina Bernocco nasce nelle Langhe nel 1922, ma cresce a Savona. Dopo la Laurea in Letteratura Inglese conseguita presso l'Università di Torino, inizia a collaborare con quotidiani locali di Genova. Le due terre, quella piemontese e quella ligure, rimarranno dei punti fermi nella sua vita e nelle sue opere letterarie. Il Piemonte è dove conosce Natalia Ginzburg, Cesare Pavese, e Beppe Fenoglio. La Liguria significa l'incontro con gli amici e maestri Adriano Grande, Camillo Sbarbaro, e il futuro marito Emilio Lagorio, responsabile del PCI nel CLN di Savona. Con lui Gina condivide i valori civili, la lotta per la Resistenza e la frequentazione di artisti e intellettuali impegnati.

La morte precoce di Emilio nel 1964 (rievocata nel romanzo del 1971, Approssimato per difetto) comporta un pesante aggravamento delle responsabilità familiari e professionali: Gina insegna e nel contempo collabora già con diversi periodici e riviste letterarie («Letterature Moderne», «L'Approdo», «Il Ponte»), ha iniziato a pubblicare i suoi primi racconti sui giornali e deve occuparsi da sola dell'educazione di due figlie.

7 Negli anni Settanta decide di trasferirsi a Milano, dove intensifica il suo lavoro giornalistico, editoriale e soprattutto letterario: pubblica nel 1977 il romanzo La spiaggia del lupo, nel 1979 Fuori scena, nel 1983 Tosca dei gatti, nel 1987 il Golfo del Paradiso, nel 1991 Tra le mura stellate, ed ancora nel 1993 Il silenzio, nel 1996 Il bastardo, un anno più tardi il romanzo autobiografico Inventario e nel 1999 L'arcadia americana. Continua ad occuparsi di critica collaborando alla realizzazione dell'Enciclopedia Europea, curando l'antologia Cultura e letteratura ligure del '900, scrivendo monografie dedicate a Fenoglio, Sbarbaro e Barile.

8 Il trasferimento a Milano si rivela determinante anche per la sua vita privata: qui incontra il suo secondo marito, l'editore Livio Garzanti. La loro sarà una lunga unione affettiva, intellettuale e professionale.

9 Negli anni Settanta Gina Lagorio è oramai affermata nella società culturale italiana. Quello che rende il suo profilo intellettuale tanto moderno è la sinergia fra i diversi settori in cui si è impegnata e i diversi registri che ha saputo modulare. Gli anni Settanta-Ottanta infatti sono gli anni in cui Gina collabora con la Radiotelevisione 
Italiana, realizzando rubriche su argomenti di cultura e di attualità, particolarmente sensibili al mondo delle donne: Singolare femminile, Parlare al femminile.

E negli stessi anni, dal 1987 al 1992, diviene deputata al Parlamento Italiano per il gruppo Sinistra Indipendente, coronando un lungo impegno civile e rappresentando $\mathrm{i}$ diritti delle donne, della famiglia e della scuola. Il ruolo televisivo e politico della Lagorio ne confermano la riconoscibilità tanto presso il pubblico colto quanto presso il più largo pubblico popolare. Gina è chiamata a parlare alle donne attraverso i più diffusi mezzi mediatici e a rappresentarle in Parlamento, il suo profilo è dunque rappresentativo della condizione femminile modernamente intesa.

Mentre i suoi romanzi ed i suoi articoli ci restituiscono la ricchezza delle sue attività culturali e del suo engagement, i documenti d'archivio restituiscono la Lagorio madre e moglie. È significativa la lettera con la quale Gina Lagorio risponde ai complimenti di Carlo Betocchi per la pubblicazione del romanzo Fuori scena:

Forse, senza quella madre che aveva fatto le elementari soltanto, ma leggeva le lettere di Leopardi, la bambina ch'ero io non sarebbe cresciuta con l'amore dei libri e forse nel momento difficile del matrimonio e della maternità, quando anche l'amore della letteratura può apparire ingiusto o non necessario, come un amore clandestino, senza l'amicizia protettiva e sorridente, stimolante ma discreta, attenta a quel che ero, nella mia domesticità ben difesa ma anche a quello che non dovevo sciupare o costringere fino a sacrificarlo, dei miei due vecchi amici, oggi io, non sarei qui a scrivere a lei, pensando anche a loro, e a tutto, a tutta la mia vita, che in qualche maniera ho riversato nel mio ultimo libro'.

La lettera risale al 24 novembre 1979. Gina Lagorio è già nota per il suo impegno letterario e civile. Quello che stupisce è il candore con il quale ammette le sue umili origini e la difficoltà, tutta propria della condizione femminile, di pacificare i doveri familiari con l'amore per la scrittura che viene definito addirittura "clandestino». Il termine «amore» da un lato simboleggia la passione di Gina per la letteratura, ma dall'altro sembra relegare la scrittura ad una pratica ricreativa e non professionale. Interessante notare che il supporto decisivo al completamento della sua opera giunse proprio da due uomini: gli amici a cui Gina fa riferimento qui e in più occasioni sono Camillo Sbarbaro e Angelo Barile, «il consigliere amico ma rigoroso, in quella sua maniera civilissima tesa all'essenziale e mai alla vanità» ${ }^{10}$.

La particolare difficoltà di coniugare il ruolo di donna di famiglia con quello di donna nelle arti è costante negli scambi epistolari con un'altra donna d'eccezione, la scrittrice e amica Alba de Céspedes. Il Centro Apice conserva le lettere inviate dalla letterata italo-spagnola a Gina Lagorio fra il 1967 e gli anni Novanta. I documenti attestano una lunga amicizia, resistita ai tanti viaggi e trasferimenti della de Céspedes fra Parigi e Cuba. Con cadenza a volte persino mensile, Gina ed Alba si scambiavano aggiornamenti sui loro progetti di lavoro, considerazioni sulle evoluzioni sociali e politiche, consigli riguardo le tendenze editoriali. Accanto ai loro discorsi più impegnati sul fronte civile $\mathrm{e}$ culturale, si possono leggere confessioni sulla loro vita privata, facezie e aneddoti di vita quotidiana, mai banali. Scrive Alba in una lettera del 7 marzo 1970:

Vorrei parlarti anche del tuo libro, così fresco, così sincero, che mi ha dato l'impressione di entrare in casa tua, di conoscere la tua famiglia. (Immagino le prime noti della Titti, e il concerto, e la tua disperazione, quel sonno arretrato, quella stanchezza che ci portiamo appresso da un giorno all'altro...) e il tuo libro, ancora una volta, ripropone il dramma di quel dualismo che è il nostro peggior nemico: la necessità di avere nella mente vari sportellini che si aprono, scattano al 
momento dato, rovesciando il cruccio, l'obbligo, l'urgenza, che ci sottrae al lavoro, o

a un'altra cosa, sempre vigili, sempre lucide e responsabili ${ }^{11}$. riserbate alle sue opere da parte della Lagorio, in veste e di amica e di critica, e soprattutto di donna, denunciando la diffusione di comportamenti maschilisti. La lettera del 26 giugno 1976 è esemplare:

Mia carissima Gina,

a sostenere le ragioni del femminismo e le ingiustizie, i pregiudizi che ancora oggi subiscono le donne basterebbe la qualità del tuo scritto sulla mia «Notte». A parte la gioia, la commozione che mi ha procurato, la soddisfazione di essere compresa in ogni intenzione, non potevo, leggendolo, impedirmi di pensare che ben pochi uomini, critici celebrati, saprebbero scrivere un articolo acuto, logico, com'è il tuo; e pochi, amando l'opera di cui parlano, saprebbero così bene mitigare il loro entusiasmo e trasmetterlo ai lettori. Ma quale quotidiano, quale rivista, ha per titolare della critica letteraria una donna? E grazie anche per aver rimesso a posto chi ha tacciato di femminile ciò che scrivo. (Femminile, sì, ma nel senso che intendiamo noi) ${ }^{13}$.

Nelle parole della scrittrice c'è una critica diretta contro l'opinione comune che associa la letteratura femminile a prodotti intimistici e psicologistici, insensibili agli eventi storici e alla sfera sociale, e difatti Alba ringrazia Gina per aver saputo leggere la sua opera con la dovuta distanza critica, smentendo coloro che potrebbero considerare il suo articolo come troppo emotivo. Al tempo stesso, le due amiche non vogliono rinnegare l'esistenza di un modo di sentire, vivere e scrivere tutto femminile: «Femminile, sì, ma nel senso che intendiamo noi». 
Le due scrittrici condividono gli stessi ideali letterari e civili, convinte che la scrittura debba alimentarsi della quotidianità piccola e grande: dalle avventure e difficoltà della sfera personale alle grandi trasformazioni della sfera collettiva.

Anche il giudizio o l'intimismo lirico fanno parte di un discorso che oltrepassa l'autobiografia già nella «stagione della prima persona», che assume piuttosto un ruolo specifico all'interno di quest'ordine: è la base del vero, il senso letterale e storico con cui fare i conti, perché - come voleva Adorno - non si dà vera vita nella falsa. Memore della distinzione tra personaggi-uomo e personaggi-particella che fu il tema di una memorabile conferenza di Giacomo Debenedetti, infatti Gina fece sua la volontà critica di trovare una chiave d'interpretazione che lasciasse spazio alla vita, tutta la vita, entrando a far parte di quella famiglia di scrittori che Magris chiamerà agrimensori del reale, curiosi delle cose e dei destini, affascinati dalle storie della vita, più imprevedibile di ogni immaginazione. I suoi personaggi dunque sono sempre 'personaggi-uomo', figure integrali che non perdono mai del tutto la loro identità, ma, al contrario, la difendono dalla frammentazione, lasciando intendere che lo scrittore non parla di sé, ma per tutti ${ }^{14}$.

Basti pensare alle strategie narrative con le quali Gina modula il loro autobiografismo: Approssimato per difetto, che l'autrice dedica al suo primo marito, appare come un diario pubblico in cui la storia del suo matrimonio con Emilio Lagorio si intreccia alla storia della Resistenza italiana. I dati privati acquistano uno spessore sociale e non sono mai gratuiti: per esempio, i loro primi incontri nel parco sono in realtà occasioni per diffondere, senza attirare sospetti, i fogli ed i giornali di opposizione al regime.

21 A questo riguardo, è significativo che Gina Lagorio scelga di parlare di alcune delle poesie scritte da Alba de Céspedes durante i moti parigini del 1968 nella propria rubrica culturale trasmessa dalla Radiotelevisione Italiana, Singolare Femminile.

Nelle lettere intercorse in quel periodo, Alba aggiorna l'amica sulle evoluzioni sociali e politiche che animano la città di Parigi e che le hanno ispirato le sue Chansons. Il 9 aprile scrive a Gina:

Grazie delle tue affettuose parole, sai con quanta spontaneità ci siamo sentite trovate e moderne e come sinceramente io ricambi il tuo affetto.

Non so quando partiranno queste righe: qui stiamo vivendo giorni appassionanti e duri. La Rue de Tournon è al centro delle rivolte: nelle ultime notti non si udivano che granate lacrimogene scoppiare e grida. Nessuno si avvicina a questo quartiere, è come un lebbrosario, ma è davvero il cuore di Parigi. Ti piacerebbe essere qui ${ }^{15}$.

Ad agosto dello stesso anno aggiunge: «Sapevo che avresti preso parte spiritualmente a ciò che accadeva a Parigi. Io, senza quasi volerlo, ho scritto delle poesie in francese, quasi un diario, in quel periodo straordinario» ${ }^{16}$. Gina presenterà alcuni dei componimenti dell'amica nel corso del suo programma radiofonico e Alba, entusiasta, considererà la trasmissione un successo non solo personale ma anche storico:

Sono rimasta commossa dall'entusiasmo per le mie Chansons che hai saputo esprimere in modo così forte e convincente... Temevo che tu avessi fatto un lavoro inutile, giacché dubitavo che la conversazione sarebbe stata accettata. È un segno confortante per il Nostro Paese, per la Rai, e una prova inconfutabile della stima che tu riscuoti ${ }^{17}$.

Il registro dei loro scambi epistolari risponde al loro particolare ideale di cultura femminista e femminile. In alcuni casi la de Céspedes, facendo tesoro delle sue esperienze all'estero, indica a Gina i nomi di donne che si stanno distinguendo nella società intellettuale internazionale, al fine di diffondere esempi positivi e combattere l'arretratezza della realtà italiana. D'altro canto, la Lagorio informa l'amica sulle evoluzioni e trasformazioni dell'editoria italiana, fornisce consigli riguardo alle case 
editrici più appropriate per pubblicare le sue opere e monitora la diffusione della letteratura italiana al di là dei confini nazionali.

In una lettera del 5 giugno 1976, Alba, dopo aver ricordato con affetto una serata trascorsa a Milano insieme a Gina, scrive:

André Pieryre de Mandiergues ha pubblicato da Gallimard un dialogo con Francine Mallet, una scrittrice e giornalista molto apprezzata e conosciuta. Io l'ho trovato molto interessante, anche per il periodo e il mondo che evoca, e anche per ciò che dice delle donne artiste. Te l'ho fatto inviare da lui personalmente. Vedi se può interessare alla Garzanti ${ }^{18}$.

Gli scambi epistolari con Alba de Céspedes ci restituiscono il ritratto di Gina Lagorio come donna moderna, affermata nel mondo editoriale e letterario non solo per i suoi romanzi, ma anche per la sua capacità nel riconoscere e diffondere nuovi modelli cultuali, più sensibili all'identità artistica femminile, con uno sguardo privilegiato alle più evolute realtà europee.

Infatti, fra i contatti d'eccezione nell'epistolario della Lagorio, non ci sono solo scrittrici ma anche politiche - come Giovanna Ioli e Cesira Fiori, «gentilissima compagna» ${ }^{19}$ con la quale ha condiviso l'esperienza della Resistenza - ed anche lettrici ed ammiratrici che trovano in Gina Lagorio una rappresentante e interlocutrice. La conservazione di queste lettere dimostra la profonda dedizione di Gina verso il suo lavoro e la grande fiducia da lei riposta nelle potenzialità della comunicazione mediatica oltre che letteraria. L'autrice trova nella dimensione civile della scrittura la soluzione a quel disagio interiore sempre presente nelle lettere fin qui rievocate.

Scrive nel suo romanzo autobiografico del 1997, Inventario:

Volevo verificare se c'era qualche necessità nel mio bisogno di dire, e soprattutto se il mio essere e sentirmi in bilico fra due mondi, quello caldo solido confortevole della mia casa e quello seducente fragile ambiguo dei libri, potesse trovare una composizione pacifica che mi salvasse dalla dissociazione. Ero giovane e pativo della mia mutabilità ai richiami delle ore, mi pareva di abitare veramente solo l'attimo, senza riconoscermi davvero in nessun luogo e in nessun presente $e^{20}$

29 Queste parole svelano che il conflitto della scrittrice non era solo tra dimensione familiare e dimensione professionale, ma anche tra ragioni personali e ragioni collettive. La scrittura deve rispondere a dei canoni non solo estetici ma anche morali: affinché la sua istanza comunicativa sia legittima deve essere socialmente utile, necessaria, non deve ridursi a semplice bisogno soggettivo.

L'apice di tale processo esistenziale e ideologico è l'approdo all'attività politica, svolta dalla Lagorio prima fra le fila della Resistenza, poi fra le pagine di giornali e riviste, ed infine nelle aule del Parlamento.

31 Nel 1987 infatti è eletta nelle liste del PCI per la circoscrizione della Ligura. Nel Centro Apice sono conservati i dattiloscritti delle dichiarazioni ufficiali pubblicate in seguito su giornali e bollettini politici. Il 9 maggio 1987, in occasione della sua elezione, Gina pronuncia queste parole:

Perché accetto:

Piemontese d'origine e ora residente a Milano, ho vissuto in Liguria gli anni che segnano nella formazione e nel destino. Studi, matrimonio, figlie, insegnamento, sono avvenuti a Savona. E soprattutto qui sono stata accanto, in anni storici, a Emilio Lagorio. Il primo manifesto di Savona libera reca la sua firma insieme a quelle degli altri membri del CNL che assunse in città i poteri di amministrazione $\mathrm{e}$ di governo il 25 aprile 1945 dopo la clandestinità. 
Non sum dignus, dovrei dire, lo so. E tuttavia, pur consapevole dei miei limiti, accetto, nel nome del padre delle mie figlie, con la speranza di corrispondere alla fiducia accordatami, in quanto persona legata alla storia della Repubblica, se non dalla militanza politica, dalla passione civile. Di questa passione, testimoniano le opere, non solo di narrativa, ma di saggistica, di giornalismo, di teatro, dai vent'anni di collaborazione al «Ponte» di Calamandrei alla commedia sul terrorismo del $1983^{21}$

Le tappe più importanti della sua storia personale appaiono irrimediabilmente intrecciate a quella della sua città. Le origini e l'appartenenza al territorio sono dei valori costanti anche nella produzione letteraria della Lagorio: territorio che non si traduce solo in semplice sfondo geografico, ma si compone di ideali, tradizioni, personaggi, come il suo primo marito Emilio. E infatti continua:

Accetto cioè in quanto intellettuale. Voglio dire che al di là delle motivazioni biografiche, il perché di questa scelta ha le sue radici nel mio mestiere di scrittrice. Chi scrive occupa una posizione ambigua sia a livello sociale, sia nell'intimo della propria esistenza e nel privato svolgersi della propria attività: se infatti il mondo sperimentato dallo scrittore nel suo lavoro è l'unico e plurisfaccettato mondo della parola, duplice è il modo di rapportarvisi. Per lo scrittore puro, la parola è il suo fine, per lo scrittore il cui fine è comunicare, la parola non è che un mezzo, sia pure il più importante, necessario, ineludibile. Chi scrive e sente il peso della responsabilità del proprio fare, non può che oscillare fra due tendenze in un certo senso contrapposte: quella dell'asservimento alla parola e al linguaggio letterario e quella che piega questa parola e questo linguaggio a un impegno di comunicazione. In questo preciso momento storico, nella confusione e nella indeterminatezza dei valori che lo costituiscono, penso sia essenziale, ritorni ad essere essenziale, affermare la propria presenza politica, il proprio esistere cioè in relazione e in comunicazione, come individuo sociale ${ }^{22}$.

La neo-eletta dichiara fin da subito il legame indissolubile fra vocazione alla scrittura e vocazione alla politica, un dovere morale che affonda le radici nella parola e nella comunicazione. La sua dichiarazione è politica e letteraria allo stesso tempo: il linguaggio deve essere investito di spessore sociale e per questo deve conservare il suo status di mezzo, di canale. Lo scrittore non deve lasciarsi sopraffare dalla facile calligrafia, come il politico non deve cedere alla vuota retorica. Difatti, nei suoi libri la Lagorio scrittrice ha sempre fuggito lo sperimentalismo formale, rimanendo ancorata ad una rappresentazione quanto più realistica. In un articolo apparso su «l'Unità» il 22 marzo 2002, Gina dichiara:

Così come spetta a chi ha la responsabilità della vita di tutti, onorare il minimo della decenza. Quella montaliana: sappiamo con certezza ciò che non siamo e ciò che non vogliamo. È troppo esigere da chi ci governa in nome di leggi democraticamente accettate almeno la decenza montaliana nella delicata, e rischiosissima, arte retorica del comunicare? ${ }^{23}$

Gina termina il suo intervento appellandosi alla partecipazione politica collettiva. «Presenza politica» è qui sinonimo di presenza nel mondo: l'uomo ha il dovere e non solo il diritto di affermare i propri valori e prendere parte attivamente ai processi di trasformazione della società. La Lagorio si dichiara sostenitrice di una più larga ma anche più responsabile democraticità, in opposizione all'indifferenza, male morale e civile. La «presenza politica» è un atto di resistenza: «[...] opporsi, ciascuno nel proprio ambito per quello che sa e può, perché non muoia nell'indifferenza dei più e nella confusione politica artatamente alimentata la capacità di pensare, in una dialettica che rispetta le idee che non si condividono ${ }^{24}$. 

verso il regime ${ }^{27}$.
Questo disegno filosofico-politico è ulteriore dimostrazione dell'estrema semplicità e umiltà di Gina Lagorio, che riconoscerà più volte di non trovarsi a suo agio fra le aule del potere. Del resto, le origini del suo impegno politico affondavano in manifestazioni autonome, atti di ribellione spontanea contro la dittatura. Gina ha sempre partecipato all'affermazione di valori etici e civili prima ancora che politici, fuggendo modelli programmatici e ignorando le dinamiche codificate all'interno dei partiti.

Durante il primo anno del suo incarico, il giornale «Sinistra Indipendente» pubblica un suo intervento dal titolo loquace: Alice nel Paese dei Deputati.

Mentre giro su me stessa come una trottola impazzita, tra chilometri di sale e di corridoi, invariabilmente perdendo l'orientamento e mentre adempio alle prime necessarie operazioni per essere del tutto onorevole, mi occupa un solo pensiero: siamo troppi, siamo troppo onorati, non è possibile una discriminazione così pesante tra chi ci ha eletto e noi che li rappresentiamo. Una legge che raddrizzi questa barca dei miracoli è non solo giusta, ma necessaria. [...]

Dalla sala della posta a quella di lettura, dalla Cit ai telefoni, dal fotografo al funzionario del tesoro, la marcia in Montecitorio ha tappe fitte e spossanti per me che ogni volta mi ritrovo, l'occhio smarrito, contro un muro in cui porte misteriose si aprono su interni sbagliati, diversi cioè da quelli cui dovevo accedere. Mi sento Alice nel paese dei deputati: stranita e stupita, ogni momento m'imbatto in qualcosa che ignoravo, e sempre il sorriso ossequioso di un commesso arriva a salvarmi ${ }^{25}$.

Mentre la Lagorio scrittrice si sente spaesata fra i palazzi del potere, la Lagorio deputata non perde di vista gli obiettivi sociali e culturali della sua missione civile:

Un atto perciò, il mio, di adesione al programma e alle liste del PCI che è insieme memoria del passato e fiducia nel rilancio di un progetto più concretamente umano, per una democrazia come effettivo tessuto della vita civile, nella quale ovviamente la mia attenzione è rivolta al confronto critico delle idee, ai problemi della scuola e all'affermazione reale dell'autonomia femminile ${ }^{26}$.

In veste di parlamentare, Gina ha partecipato attivamente ai dibattiti su questioni cruciali per la storia della società italiana. Interessante è la sua posizione riguardo all'insegnamento di religione nelle scuole pubbliche. Dopo che il Concordato del 1984 aveva eliminato l'obbligatorietà dell'ora di religione nelle scuole italiane, un'accesa protesta proveniente dall'ambiente cattolico ha costretto il Parlamento a ridiscutere i termini della facoltatività. Gina Lagorio, da donna cattolica ma intellettuale laica, si appella agli ideali progressisti e liberali di Piero Calamandrei in difesa dell'autonomia culturale del Paese. Nella sua dichiarazione dell'ottobre 1987, Gina dimostra di saper scindere criticamente fede personale da interesse comune:

Ora, se la nostra storia e la nostra cultura hanno trovato nel cristianesimo lievito ed anima, hanno avuto anche nel potere temporale della Chiesa cattolica un blocco costante ed ostinato, una croce da portare tanto più tormentosa quanto più gli spiriti erano alti e intimamente religiosi. Eanche adesso, non di un'ora di insegnamento soltanto si tratta, ma di qualcosa di ben più significante.

Negare i diritti dello Stato in nome della Chiesa e viceversa, a questo punto, non è che un esercizio di parata attraverso cui far passare ben altro che una situazione didattica di orario. Cresce la marea degli indifferenti: il materiale umano migliore perché «la repubblica pontificia», che allarmava Piero Calamandrei in anni che sembrano lontanissimi, a tali non sono, scivoli dolcemente, senza traumi apparenti,

Un altro dei dibattiti al quale la Lagorio ha partecipato con più passione è quello scoppiato in seguito alla legge sull'aborto. Dopo dieci anni dalla sua approvazione, gli opposti schieramenti politici e ideologici si interrogavano ancora sulla validità morale e 
sociale di tale legge e sui suoi effetti. L'atteggiamento di Gina Lagorio sarà oggetto di critica da parte dei movimenti extra-parlamentari:

Si è parlato di violenza, di stupro e di aborto, proprio mentre stava ratificandosi da noi il primo come reato contro la persona pur fra polemiche accese, e sul secondo siamo in clima di crociata. L'aborto è dolore, è trauma; che questa sofferenza sia contenuta finché nuovi modelli educativi e civili l'avranno eliminata. Ora valga la legge, senza la quale le donne più sfruttate ed emarginate lo sarebbero ancora di più. Ma com'è triste che ci si scanni ancora per l'ideologia, quando solo la sofferenza delle donne è il prezzo di ciò che si dibatte ${ }^{28}$.

Le sue parole sembrano ad una prima lettura troppo timide e sommesse rispetto al suo profilo di donna moderna e progressista. La posizione della nostra scrittrice, però, non è dettata da bigottismo o remore religiose, bensì da un sincero pragmatismo, superiore alle leggi di partito e noncurante dei facili consensi o dissensi provenienti dalle piazze. La Lagorio riconosce la necessità di legalizzare una pratica che negli anni precedenti, relegata alla clandestinità e quindi a condizioni igienico-sanitarie disumane, aveva provocato la morte di migliaia di donne, ma limita l'utilità sociale della legge ai Paesi meno sviluppati, dove effettive condizioni civili ed economiche richiedono strategie radicali per tutelare le donne. Il discorso è diverso per i Paesi come l'Italia, dove la condizione di benessere sociale e lo stato di diritto dovrebbero assicurare al genere femminile un più facile accesso ai contraccettivi, una corretta educazione sessuale ed una maggiore tutela dalle violenze maschili, all'interno e fuori dalle mura domestiche. Ricordiamo che solo nel 1981 il Parlamento italiano ha abolito il delitto d'onore e che solo nel 1996 riconoscerà la violenza verso la donna un reato contro la persona e non più contro la morale pubblica. Agli occhi della Lagorio, l'aborto è una indiscutibile conquista ma non è il fine ultimo della lotta femminile e femminista, e i dibattiti ideologici non devono oscurare aspetti ancora trascurati dalla Costituzione. La legge del 1978 era stata il frutto di una lunga contrattazione parlamentare, dalla quale non tutti i partiti uscirono soddisfatti, primo fra tutti quello Radicale che si era fatto portavoce delle istanze femministe. Negli anni a seguire, gli oppositori cattolici tentarono di farla abrogare mentre movimenti più radicali avanzarono proposte verso una maggiore liberalizzazione.

Essa consentiva l'interruzione volontaria della gravidanza a spese dello Stato entro i primi novanta giorni. L'aborto presso strutture private rimaneva illegale. La gravidanza o il parto dovevano comportare un serio pericolo per la salute fisica o psichica della donna, tenendo conto delle sue condizioni economiche, sociali e familiari e delle circostanze in cui era avvenuto il concepimento (per esempio lo stupro). Nella maggior parte dei casi, le donne dovevano attendere che fosse trascorso un periodo di riflessione di sette giorni prima di procedere. [...] Il personale sanitario aveva la facoltà di dichiarare la propria «obiezione di coscienza». Moltissimi lo fecero, i 72\% dei medici nel 1979, soprattutto al Sud e nel Nordest a forte influenza cattolica ${ }^{29}$.

Secondo l'opinione della Lagorio, la questione dell'aborto appare troppo superficialmente legata alla violenza carnale. Gina, invece, considera abuso non solo lo stupro, ma anche le pressioni esercitate dalla comunità religiosa e dal perbenismo diffuso che obbligano moralmente una donna a sposare il padre biologico di suo figlio. La deputata cerca di aprire il dibattito politico-ideologico ad una prospettiva nuova, ancora non tollerata in Italia negli anni Settanta, nonostante i moti di emancipazione. Del resto, in veste di scrittrice aveva già chiarito la sua posizione in uno dei suoi romanzi più famosi. Ne La spiaggia del lupo, Lagorio racconta la Bildung di Angela, prima bambina curiosa e fantasiosa che vive le sue avventure in un piccolo paese della Liguria, 
poi giovane donna indipendente e spregiudicata, che si trasferisce nella grande Milano. Appena diciottenne, la protagonista conosce Vladi, un ragazzo affascinante che si trova ad Oneglia per le vacanze estive e con il quale intrattiene un'appassionata relazione. Qualche tempo dopo la sua partenza, Angela scopre di essere rimasta incinta e lui le confessa di essersi sposato. Dopo aver comunque scelto di raggiungerlo a Milano e lottare per la loro relazione, Angela resta ben presto delusa dalle esitazioni di Vladi, capisce di non amarlo e di non voler rinunciare al bambino. Decide così di affrontare la gravidanza e la maternità da sola, sfidando le paure e i pregiudizi. Angela riconoscerà alla figlia la libertà che la piccola provincia ha invece sempre negato a lei stessa:

Ci pensava ora, mentre Angela correva in mare e sul filo dell'acqua dondolavano i gabbiani: anche Angela aveva due ali in corpo, contratte, ma quando come ora allargava le braccia prima di raccoglierle unite sulla testa in un tuffo perfetto, e tagliava il mare senza increspatura di schiume, sua figlia le sembrava una creatura non sua, prima uccello e poi pesce, di quelli che saltano, come i salmoni e non sai se appartengono all'acqua o al cielo ${ }^{30}$.

Il fitto intreccio di consonanze tra vocazione civile e scrittura letteraria, tra valori politici e principi personali, tra dimensione privata ed impegno pubblico restituisce una ricca antologia della vita di Gina Lagorio. Ad una lettura complessiva, il disagio interiore, costante nei suoi romanzi e nelle sue confessioni amicali, si rivela sintomo di profonda coerenza artistica e morale. La figura di Gina, divisa e condivisa fra molteplici attività, si ricompone in un profilo quanto più diversificato, tanto più organico di donna moderna.

\section{NOTE}

1. Restano interdetti al genere femminile gli uffici presso: Polizia (fino alla legge n. 121 del 1981), Forze Armate, Carabinieri e Guardia di Finanza (fino alla legge n. 380 del 1999).

2. Archivi della Parola, dell'Immagine e della Comunicazione Editoriale dell'Università degli Studi di Milano [APICE], Archivio Gina Lagorio (da ora in avanti APICE G. L.) serie 3, fasc. 97.

3. APICE G. L., serie 3, fasc. 405.

4. APICE G. L., serie 3, fasc. 511.

5. APICE G. L., serie 3, fasc. 528.

6. APICE G. L., serie 3, fasc. 704.

7. APICE G. L., serie 3, fasc. 631.

8. APICE G. L., serie 3, fasc. 990.

9. APICE G. L., serie 3, fasc. 116.

10. Ibid.

11. APICE G. L., serie 3, fasc. 405

12. Ibid.

13. Ibid.

14. G. Ioli, Dal «Ciclone» a «Càpita»: un campionario del mondo, in L. Clerici (a cura di), Gina Lagorio. La scrittura tra arte e vita, Roma, Edizioni di storia e letteratura, 2010, pp. 7-14.

15. APICE G. L., serie 3, fasc. 405. 
16. Ibid.

17. Ibid.

18. Ibid.

19. L'appellativo ricorre in più lettere conservate all'interno dell'archivio, APICE G. L., serie 3, fasc. 528.

20. G. Lagorio, Inventario, Milano, Rizzoli, 1997.

21. APICE G. L., serie 2, fasc. 5.

22. Ibid.

23. Ora in G. Lagorio, Parlavamo del futuro, Melampo, 2011, p. 247.

24. Ibid.

25. APICE G. L., serie 2, fasc. 21.

26. Ibid.

27. APICE G. L., serie 2, fasc. 11.

28. APICE G. L., serie 2, fasc. 29.

29. P. Willson, Italiane. Biografia del Novecento, Bari-Roma, Laterza, 2010, p. 288.

30. G. Lagorio, La spiaggia del lupo, Milano, Garzanti, 1977.

\section{RIASSUNTI}

Impegnata fin dalla sua gioventù sul fronte politico e culturale, Gina Lagorio ha rappresentato con efficacia le conquiste e le contraddizioni proprie della donna moderna. Il Centro Apice (Università degli Studi di Milano) conserva l'intero archivio della scrittrice: appunti, articoli per giornali, dattiloscritti dei suoi interventi parlamentari, bozze di opere saggistiche e narrative, un ricco epistolario. Le fonti si rivelano preziose per più di un motivo. Dall'analisi delle lettere intercorse con un'altra donna d'eccezione, Alba de Céspedes, emergono non solo riflessioni sull'attualità culturale e politica, ma anche la difficoltà di coniugare lavoro intellettuale e doveri familiari. Gli interventi pubblicati sui giornali e tenuti dalla Lagorio nelle aule del Parlamento contribuiscono alla ricostruzione di tappe cruciali del percorso di emancipazione femminile nel secondo Novecento.

Strongly engaged since her youth on both the political and cultural fronts, Gina Lagorio strongly embodied the victories and contradictions of the modern woman. The Centro Apice (Università degli Studi di Milano) houses all her archives: notes, press articles, transcripts of her parliamentary speeches, drafts of her essays and literary works, and a rich correspondence. The sources are precious for several reasons. The letters she exchanged with another exceptional woman, Alba de Céspedes, not only contain interesting reflections on cultural and political news, they also show the difficulty of reconciling intellectual work with family duties. The articles she published in the newspapers and her interventions from the benches of parliament help us to reconstruct the crucial stages in the journey of women emancipation in the second half of the 20th century.

Engagée depuis sa jeunesse sur les fronts politique et culturel, Gina Lagorio a représenté avec force les conquêtes et les contradictions propres à la femme moderne. Le Centro Apice (Università degli Studi di Milano) conserve toutes les archives de l'écrivaine : notes, articles de presse, retranscriptions de ses interventions parlementaires, ébauches de ses essais et de ses 
œuvres littéraires, ainsi qu'une riche correspondance. Les sources se révèlent précieuses pour plusieurs raisons. De l'analyse des lettres échangées avec une autre femme d'exception, Alba de Céspedes, émergent non seulement des réflexions sur l'actualité culturelle et politique, mais aussi la difficulté de conjuguer travail intellectuel et devoirs familiaux. Les articles qu'elle a publiés dans les journaux ainsi que ses interventions depuis les bancs du parlement contribuent à la reconstruction d'étapes cruciales de l'émancipation féminine dans la deuxième moitié du $\mathrm{xx}^{\mathrm{e}}$ siècle.

\section{INDICE}

Keywords : Gina Lagorio, Emilio Lagorio, Garzanti, archives, correspondences, women emancipation, second half of the 20th century, feminist movement, abortion

Parole chiave : Gina Lagorio, Garzanti, politica, archivi editoriali, donne parlamentari, Sinistra parlamentare, PCI, Emilio Lagorio, letteratura italiana contemporanea, movimento femminista, aborto

Mots-clés : Gina Lagorio, Garzanti, archives, correspondances, Parti communiste italien, travail intellectuel, émancipation féminine, avortement

\section{AUTORE \\ MARIE LOUISE CRIPPA \\ Università degli Studi di Milano \\ marie.crippa@unimi.it}

